\title{
Nanog/NFATc1/Osterix signaling pathway-mediated promotion of bone formation at the tendon-bone interface after ACL reconstruction with De-BMSCs transplantation
}

Kai Tie

Wuhan University Zhongnan Hospital

Jinghang Cai

Wuhan University Zhongnan Hospital

Jun Qin

Wuhan University Zhongnan Hospital

Hao Xiao

Wuhan University Zhongnan Hospital

Yangfan Shangguan

Wuhan University Zhongnan Hospital

Hui Wang

Wuhan University School of Basic Medical Sciences

Liaobin Chen ( $\square$ lbchen@whu.edu.cn )

Wuhan University Zhongnan Hospital

\section{Research}

Keywords: dedifferentiation, BMSCs, osteogenic differentiation, anterior cruciate ligament, tendon-bone healing

Posted Date: June 16th, 2021

DOI: https://doi.org/10.21203/rs.3.rs-612939/v1

License: (c) (i) This work is licensed under a Creative Commons Attribution 4.0 International License. Read Full License

Version of Record: A version of this preprint was published at Stem Cell Research \& Therapy on November 14th, 2021. See the published version at https://doi.org/10.1186/s13287-021-02643-9. 


\section{Abstract}

Background: Bone formation plays an important role in early tendon-bone healing after anterior cruciate ligament reconstruction (ACLR). Dedifferentiated osteogenic bone marrow mesenchymal stem cells (DeBMSCs) have enhanced osteogenic potential. This study aimed to investigate the effect of De-BMSCs transplantation on the promotion of bone formation at the tendon-bone interface after ACLR and to further explore the molecular mechanism of the enhanced osteogenic potential of De-BMSCs.

Methods: BMSCs from the femurs and tibias of New Zealand White rabbits were subjected to osteogenic induction and then cultured in medium without osteogenic factors; the obtained cell population was termed De-BMSCs. De-BMSCs were induced to undergo osteo-, chondro- and adipo-differentiation in vitro to examine the characteristics of primitive stem cells. An ACLR model with a semitendinosus tendon was established in rabbits, and the animals were divided into a control group, BMSCs group and De-BMSCs group. At 12 weeks after surgery, the rabbits in each group were sacrificed to evaluate tendon-bone healing by histologic staining, micro-computed tomography (micro-CT) examination, and biomechanical testing. During osteogenic differentiation of De-BMSCs, an siRNA targeting nuclear factor of activated T cells 1 (NFATc1) was used to verify the molecular mechanism of the enhanced osteogenic potential of De-BMSCs.

Results: De-BMSCs exhibited some properties similar to BMSCs, including multiple differentiation potential and cell surface markers. Bone formation at the tendon-bone interface in the De-BMSCs group was significantly increased, and biomechanical strength was significantly improved. During the osteogenic differentiation of De-BMSCs, the expression of Nanog and NFATc1 was synergistically increased, which promoted the interaction of NFATc1 and Osterix, resulting in increased expression of osteoblast marker genes such as COL1A, OCN, and OPN.

Conclusions: De-BMSCs transplantation could promote bone formation at the tendon-bone interface after ACLR and improve the biomechanical strength of the reconstruction. The Nanog/NFATc1/Osterix signaling pathway mediated the enhanced osteogenic differentiation efficiency of De-BMSCs.

\section{Background}

The anterior cruciate ligament (ACL) is one of the most important stable structures of the knee joint, and the meniscus and cartilage are easily injured after ACL rupture, predisposing the knee to early degenerative changes [1]. Similar to that of the articular cartilage and meniscus, the healing potential of the ACL is extremely poor [2], and reconstruction surgery is often required. Anterior cruciate ligament reconstruction (ACLR) can restore knee stability and prevent further damage to the injured knee $[3,4]$. ACLR has been widely used in clinical practice, and the incidence of ACLR has significantly increased over time [5]. Although tendon grafts, including hamstring and patellar tendon grafts, have been used in ACLR for decades with satisfactory long-term clinical outcomes [6,7], there are still some cases that require revision surgery, which is related to poor tendon-bone healing after reconstruction [8]. The success 
of ACLR largely depends on the biological healing between the graft and bone tunnel, and ideal ACLR using tendon grafts requires biological healing of the bone-to-tendon interface between the host bone and transplanted tendon. However, problems such as the relatively long healing time between tendon and bone and insufficient early biomechanical strength have not been fundamentally resolved. Therefore, promoting tendon-bone healing after ACL reconstruction is still an urgent problem to be solved.

To enhance the bone graft healing process, biological augmentation techniques, such as transplantation of bone marrow stem cells (BMSCs) [9], platelet-rich plasma (PRP) injection [10], growth factor and gene transfer [11], have been applied in ACLR in recent years. It was reported that radiolabeled BMSCs were evenly distributed at the tendon-bone interface during the healing process when they were implanted into the tendon-bone interface after ACL reconstruction [12], suggesting that BMSCs might play an important role in promoting tendon-to-bone tunnel healing. In view of the role of BMSCs in tendon-bone healing and their self-renewal and multi-differentiation potential, stem cell transplantation is considered to be a promising method to promote tendon-bone healing [13], and clinical application has been attempted [14]. Bone formation plays an important role in early tendon-bone healing [15], and the biomechanical strength of the tendon-bone connection is related to the bone mass and mineralization of regenerative tissue at the tendon-bone interface [16], implying that new bone formation in the bone tunnel is essential for early healing between bone and tendon. Previous studies using BMSCs to promote tendon-bone healing showed that transplantation of BMSCs could promote direct tendon-bone healing to some extent $[9,17,18]$, but new bone formation was insufficient at the tendon-bone interface.

Dedifferentiation refers to the transformation of cells from a given differentiated state to a less differentiated or stem cell-like state and leads to reacquisition of pluripotency; dedifferentiation is a cellular process associated with reentry into the cell cycle, trans/redifferentiation, or tissue regeneration [19]. Dedifferentiated cells possess certain characteristics of primitive stem cells, and the efficiency of redifferentiation is significantly improved. Dedifferentiation also occurs during the process of directed differentiation of stem cells [20]. Studies focused on the differentiation and dedifferentiation of BMSCs found that BMSCs could be reprogrammed in vitro via neuronal differentiation and dedifferentiation, with enhanced therapeutic efficacy [21]. During the process of osteogenic differentiation of BMSCs, BMSCs could be dedifferentiated after removing the induction conditions, and the resulting cells were termed dedifferentiated osteogenic BMSCs (De-BMSCs). De-BMSCs have improved osteogenic potential in vitro and exhibit great superiority in ectopic bone formation in vivo [21区22]. These studies, which focused on De-BMSCs, provided new insights for stem cell transplantation. Because of the essential role of bone formation at the tendon-bone interface in early tendon-bone healing, De-BMSCs implantation at the tendon-bone interface could theoretically differentiate into more osteoblasts and osteocytes during the tendon-bone healing procedure, increase bone growth in the bone tunnel and promote early tendon-bone healing.

Although the osteogenic effect of De-BMSCs is significantly enhanced, its molecular mechanism is still unclear. Nanog is a newly reported transcription factor that is expressed in primordial germ cells and embryonic stem cells and plays a key role in maintaining the self-proliferation and undifferentiated state 
of stem cells [23]. It selectively inhibits or promotes gene expression by binding to the regulatory regions of target genes. The expression of Nanog in De-BMSCs was significantly increased [20]. Osterix is an osteoblast-specific transcription factor belonging to the SP/KLF family [24]; it is also the key transcription factor for osteogenic differentiation and can induce the expression of many mature osteoblast marker genes [25] and play a vital role in the maintenance of bone formation [26]. Nuclear factor of activated Tcells $\mathrm{c} 1$ (NFATc1) is an important transcription factor for osteoclast formation [27], and it regulates the differentiation of osteoclasts. It also plays an essential role in bone formation and can combine with Osterix to form a complex and cooperatively control osteoblastic bone formation [28]. Nanog could upregulate the expression of NFATC1 during the osteogenic differentiation of stem cells, thereby promoting osteogenic differentiation [29]. Therefore, we speculated that the Nanog/NFATc1/Osterix signaling pathway might mediate the significant enhancement of osteogenic differentiation efficiency in De-BMSCs.

In the current study, we confirmed the effect of De-BMSCs transplantation to promote bone formation at the tendon-bone interface after ACLR and further explored the molecular mechanism underlying the enhanced osteogenic differentiation efficiency of De-BMSCs.

\section{Methods}

The animal experiments in this study were performed in accordance with the Guide for the Care and Use of Laboratory Animals of the US National Institutes of Health (NIH) and performed in the Center for Animal Experiments of Zhongnan Hospital of Wuhan University. The experimental design was approved by the Committee on the Ethics of Animal Experiments of the Wuhan University School of Medicine.

\section{Culture of rabbit BMSCs}

The isolation and culture of rabbit BMSCs from the tibias and femurs of 3-week-old New England white rabbits were performed as described previously [11]. In brief, the rabbits were anesthetized and sterilized using $75 \%$ ethanol for 15 min before surgery. The femurs and tibias were harvested, and the metaphysis of the bones was dissected under sterile conditions. Bone marrow cells were collected by flushing the cavity of the femurs and tibias with Dulbecco's modified Eagle's medium (DMEM)/F12 medium supplemented with 10\% fetal bovine serum (Gibco, Carlsbad, CA, USA), 50 mg/ml L-ascorbic acid (SigmaAldrich), $1 \%$ glutamine (Sigma-Aldrich), and $100 \mathrm{mg} / \mathrm{ml}$ streptomycin and penicillin (Sigma-Aldrich). The flushed liquid was centrifuged at $1200 \mathrm{rpm}$ for $8 \mathrm{~min}$. The supernatants were discarded, and the cell pellets were resuspended in culture medium, expanded in T-25 flasks (Cyagen Biosciences, Santa Clara, CA, USA) with DMEM/F12 medium and incubated at $37^{\circ} \mathrm{C}$ with $5 \% \mathrm{CO}_{2}$. The medium was changed every 3 days. When the cells reached $70 \%-80 \%$ confluence, the adherent cells were trypsinized, harvested, and expanded. Cells that had undergone three passages were used in subsequent experiments.

\section{Preparation of De-BMSCs}


To obtain De-BMSCs, according to a previous study [22], BMSCs at p3 were transferred to osteogenic induction (DMEM)/F12 medium containing $1 \mathrm{nM}$ dexamethasone, $50 \mu \mathrm{M}$ ascorbic acid, and $20 \mathrm{mM} \beta$ glycerophosphate (all from Sigma-Aldrich) for 7 days. After osteogenic induction, the BMSCs were cultured in DMEM/F12 medium without inducible factors. To achieve dedifferentiation, dedifferentiation culture with DMEM/F12 medium was performed for 3,7 , and 10 days, and then osteogenic differentiation was performed again for 14 days. The expression of osteoblast marker genes was measured to determine the best dedifferentiation culture time for De-BMSCs, which would be transplanted at the tendon-bone interface after ACLR. The De-BMSCs were osteogenically differentiated for $24 \mathrm{~h}$ for investigation of the molecular mechanism.

\section{Flow cytometry (FCM) analysis of BMSCs and De-BMSCs}

BMSCs and De-BMSCs were trypsinized and harvested and then incubated with fluorochrome-conjugated primary antibodies against CD29, CD34, CD44, and CD45 or the corresponding isotype control (BD Biosciences, USA) at $4^{\circ} \mathrm{C}$ for 30 minutes. The stained cells were immediately detected using flow cytometry (BD Biosciences, USA).

\section{Multilineage differentiation of BMSCs and De-BMSCs}

For osteogenic differentiation, BMSCs and De-BMSCs were cultured in DMEM/F12 medium containing 1 nM dexamethasone, $50 \mu \mathrm{M}$ ascorbic acid, and $20 \mathrm{mM}$ ß-glycerophosphate (all from Sigma-Aldrich) for 14 days. The mineralization of BMSCs and De-BMSCs was assessed by Alizarin red S staining and alkaline phosphatase (ALP) staining. For Alizarin red S staining, BMSCs and De-BMSCs were washed with PBS, fixed with $70 \%$ ethanol for 10 min, and stained with 0.5\% Alizarin red S (pH 4.1; Sigma, St. Louis, MO) for 5 min. ALP staining was performed with a BCIP/NBT alkaline phosphatase color development kit (Beyotime Institute of Biotechnology, Haimen, China) according to the manufacturer's instructions.

For adipogenic differentiation, BMSCs and De-BMSCs were plated in a 6-well culture plate and cultured with DMEM/F12 medium containing $1 \mu \mathrm{M}$ dexamethasone, $10 \mu \mathrm{g} / \mathrm{mL}$ insulin, $0.5 \mathrm{mM}$ 3-isobutyl-1methylxanthine, $0.2 \mathrm{mM}$ indomethacin (all from Sigma-Aldrich, USA) and 10\% FBS for 14 days [31]. Then, the cells were fixed with $70 \%$ ethanol for 10 minutes and stained with $0.3 \%$ fresh oil red 0 solution (Sigma-Aldrich) for 10 mins.

For chondrogenic differentiation, BMSCs and De-BMSCs were generated following the method described in our previous work [31]. The cells were trypsinized and washed and then centrifuged at $1000 \mathrm{rpm}$ for 5 min in a $15 \mathrm{~mL}$ polypropylene tube. The isolated cells were suspended at a concentration of $6 \times 10^{6}$ cells $/ \mathrm{ml}$ in $1.25 \%$ alginate (Sigma-Aldrich) in $0.15 \mathrm{M} \mathrm{NaCl}$, and then the cell suspension was slowly dropped into a $102 \mathrm{mM} \mathrm{CaCl}_{2}$ solution. The beads were cultured in six-well plates with $5 \% 02$ with DMEM/F12 medium containing 1\% insulin, transferrin and selenium (ITS) (Sigma-Aldrich), $100 \mathrm{nM}$ dexamethasone (Sigma-Aldrich) and $10 \mathrm{ng} / \mathrm{ml}$ transforming growth factor- $\beta 1$ (TGF- $\beta 1$ ) (Pepro Tech Rocky Hill, NJ, USA). On day 28 , the beads were fixed in $4 \%$ paraformaldehyde for $2 \mathrm{~h}$ at room 
temperature, dehydrated in serial ethanol dilutions, and embedded in paraffin blocks. Safranin 0 staining was performed on paraffin sections of the beads.

\section{Animal study}

Healthy New England Rabbits weighing 2.5-3.0 kg were purchased from the Experimental Center of the Hubei Medical Scientific Academy (Hubei, China). The rabbits were randomly distributed into the control, BMSC and De-BMSC groups ( $\mathrm{n}=8$ for each group). BMSCs and De-BMSCs were harvested 4 weeks prior to the experiment. The mixture containing BMSCs or De-BMSCs $\left(1 \times 10^{7}\right.$ cells $\left./ \mathrm{ml}\right)$ and $1.25 \%$ alginate in $0.15 \mathrm{M}$ saline was then transplanted at the tendon-bone interface of the rabbits in the three groups with a microsyringe. After ACLR, in the control group, only alginate gel was injected into the tendon-bone interface; in the BMSC group, alginate mixed with BMSCs was transplanted at the tendon-bone interface; and in the De-BMSC group, alginate mixed with De-BMSCs was transplanted at the tendon-bone interface.

The ACLR model in these three groups was established following the method described by Liu et al. [32]. In brief, anesthesia was performed via ear vein injection of $30 \mathrm{mg} / \mathrm{kg} 3 \%$ pentobarbital. In each case, both knees were shaved, disinfected and draped. A $4.0 \mathrm{~cm}$ medial parapatellar incision was made, the semitendinosus tendon was exposed and harvested at its proximal musculotendinous junction, and the tibial insertion of the tendon was preserved. The free end of the tendon was weaved with 3-0 Ethibond sutures (Johnson \& Johnson) for traction. The capsule was opened from the medial side, the patellae were laterally dislocated, and the original ACL was exposed and removed. The tibial and femoral tunnels were created with a $2.5-\mathrm{mm}$ drill at the footprint of the native ACL. The tunnels were irrigated with normal saline, and the weaved semitendinosus tendon was advanced through the tibial and femoral tunnels. When the tendon passed through the femoral tunnel, the free end of the tendon was sutured to the periosteum using 2-0 Ethibond (Ethicon, Somerville, $\mathrm{NJ}$ ) in a standard fashion. BMSCs or De-BMSCs immobilized in alginate were evenly injected into the interface between the tendon and tunnel. Following implantation, the patellar retinaculum and overlying soft tissues were closed in layers. The rabbits were allowed to move their knee joints freely in their cages without restriction. An intraperitoneal injection of $4 \times 10^{5} \mathrm{U}$ of penicillin (Harbin Pharmaceutical Group, Shanghai, China) was administered immediately after implantation and once daily for 3 days. Knee samples were harvested from each group at 12 weeks postoperatively for further analysis.

\section{Histologic analysis and immunohistochemistry}

The soft tissues were dissected from the knee samples, and the grafts were left intact. The knee samples were fixed in $10 \%$ formalin, decalcified in EDTA for 3 weeks, and then embedded in paraffin. Five-mmthick sections were cut longitudinal to the bony tunnels. The slides were stained using hematoxylin and eosin. The tendon-bone interface at a depth of $5 \mathrm{~mm}$ from the joint surface was evaluated. The expression of osteoblast marker genes, such as COL1A, OCN, OPN, and Nanog/NFATc1/Osterix signaling pathway components, at the tendon-bone interface was analyzed by immunohistological staining. After antigen retrieval by boiling the samples in sodium citrate buffer, the sections were blocked in serum for 
30 min, followed by incubation with the primary antibody in a humidified chamber at $4^{\circ} \mathrm{C}$ overnight. A biotinylated secondary antibody was added for $30 \mathrm{~min}$ on day 2, followed by an avidin-biotinylated horseradish peroxidase complex, according to the manufacturer's directions. Finally, peroxidase activity was revealed by immersion in DAB substrate. The following primary antibodies were used: rabbit antiNanog, anti-NFATC1, anti-Osterix, anti-OPN, anti-OCN, and anti-COL1A1 antibodies (all from Abcam, Cambridge, MA, USA). To characterize changes in immunostaining, the mean optical densities (MODs) were obtained from 10 areas of articular cartilage from 5 separate samples.

\section{Micro-CT analysis}

Micro-CT was performed to assess the bone mass and density of newly formed mineralized tissue inside the bone tunnels. The knee samples were harvested and carefully dissected around soft tissues, with only the bone and the graft preserved. The samples were scanned by micro-CT ( $\mu \mathrm{CT}-40$, Scanco Medical, Brüttisellen, Switzerland) with the parameters set at $70 \mathrm{kV}$ and $114 \mu \mathrm{A}$ [33]. Images were obtained to assess multiple sections of the bone tunnel. Three-dimensional image reconstruction, bone tunnel area measurement, and bone volume/total volume (BV/TV) ratio analysis in the region of interest were performed. The areas of the vertical plane across the axis of the bone tunnel were measured at a depth of $5 \mathrm{~mm}$ from the tibial joint surface. Each area was measured three times with image analysis software (ImageJ; National Institutes of Health), and the average value was used for analysis. The region of interest was cylinder shaped, $4.0 \mathrm{~mm}$ in diameter, and $4 \mathrm{~mm}$ in length.

\section{Biomechanics analysis}

Knee samples with the femur and tibia kept at a length of $50 \mathrm{~mm}$ from the joint were harvested and immediately frozen at $-80^{\circ} \mathrm{C}$ until testing. Before testing, the specimen was thawed overnight at room temperature. The femur-ACL graft-tibia complex was separated by resecting the attached soft tissue, and the tibial insertion of the semitendinosus tendon was also cut. Biomechanical testing was performed using a material testing machine (805, Instron Co., Norwood, MA, USA). The complex was fixed between the U-shaped clamps with $45^{\circ}$ of knee flexion to ensure that the pulling force was parallel to the axis of the graft. The samples were preloaded with a static preload of $1 \mathrm{~N}$ for 5 minutes and then underwent the ultimate failure load test at an elongation rate of $5 \mathrm{~mm} / \mathrm{min}$. The load-deformation curve was recorded. The ultimate failure load and stiffness were determined from the load-displacement curve.

\section{RNA interference}

siRNAs for NFATc1 (NFATc1-rabbit-1582) were purchased from Gene Pharma (Shanghai Gene Pharma Co.). The scramble sense siRNA targeted the sequence 5'-GCCCGUAUGAGCUUCGCAUTT-3', and the scramble anti-sense siRNA targeted the sequence 5'-AUGCGAAGCUCAUACGGGCTT-3'. In brief, De-BMSCs were plated to obtain $70-80 \%$ confluence in six-well plates and transfected with si-NFATc1 using Lipofectamine 2000 (Invitrogen, Carlsbad, CA, USA), negative control siRNA or Lipofectamine 2000 only. After $6 \mathrm{~h}$ of transfection, the medium was replaced with fresh medium. The expression of NFATc1 was detected using RT-qPCR and Western blotting. 


\section{Reverse transcription and real-time quantitative PCR}

To evaluate the osteogenic differentiation potential of BMSCs and De-BMSCs, gene expression at the mRNA level was examined. Total RNA from regenerated tissues and BMSCs or De-BMSCs was extracted using TRIzol (Invitrogen) reagent following the manufacturer's protocol. The RNA was reverse transcribed using a first-strand cDNA synthesis kit. The cDNA was amplified using a one-step polymerase chain reaction (RT-PCR) reaction. The relative mRNA expression levels of Sox 9 and Col2A1 were normalized to the level of glyceraldehyde 3-phosphate dehydrogenase (GAPDH). The rabbit primer sequences and annealing temperatures used are shown in Table 1.

\section{Table 1}

Primers used for qPCR

\begin{tabular}{lllc}
\hline Gene & \multicolumn{1}{c}{ Forward primer } & \multicolumn{1}{c}{ Reverse primer } & Annealing \\
\hline GAPDH & CTCAAGATTGTCAGCAACGCA & TTGGGGGTGGGCACACGGAAG & $55^{\circ} \mathrm{C}$ \\
Nanog & CCTGTGATTTGTGGGCCTGA & CTCTGCAGAAGTGGGTTGTTTG & $55^{\circ} \mathrm{C}$ \\
OCN & GCCCTCACTCTTGTCGCCC & GGCTCGCTTCACCACCTCG & $55^{\circ} \mathrm{C}$ \\
Col1A1 & GCCATCAAGGTCTTCTGCG & GAACTGGAAGCCATCGGTC & $55^{\circ} \mathrm{C}$ \\
NFATc1 & CGTTCTCTCCAACACCAAGG & CTTCTCCACAAGGGGCAGTT & $55^{\circ} \mathrm{C}$ \\
Osterix & TCAACCTCCACTGAACCCC & CCTGGTTGTAGGAGGTGGGG & $55^{\circ} \mathrm{C}$ \\
OPN & CCTGGTTGTAGGAGGTGGGG & AGGACATAGCATTCTGCGGTG & $55^{\circ} \mathrm{C}$ \\
\hline
\end{tabular}

Abbreviation: GAPDH, glyceraldehyde 3-phosphate dehydrogenase; OCN, osteocalcin; COL1A1, $\alpha 1$ chain of type I collagen; NFATc1, nuclear factor of activated T-cells c1; OPN, osteopontin

\section{Western blotting}

To obtain total protein, the cells were harvested and dissolved in RIPA (Beyotime, Nanjing, China) buffer. The protein concentrations were determined with a BCA protein assay kit. Equal amounts of protein lysates (40 mg/lane) were loaded and resolved by $10 \%$ sodium-dodecyl sulfate-polyacrylamide gel electrophoresis (SDS-PAGE) (Sigma-Aldrich) and then transferred to nitrocellulose filters and probed with rabbit anti-Nanog, anti-NFATC1, anti-Osterix, anti-OPN, anti-OCN, and anti-COL1A1 antibodies at $4^{\circ} \mathrm{C}$ overnight. After incubation with a horseradish peroxidase-conjugated secondary antibody (Santa Cruz Biotechnology), the blots were developed by enhanced chemiluminescence following the manufacturer's protocol and were visualized by exposure to a Fusion FX system (VilberLourmat, Marne-la-Vallee, France). The protein amounts in electrophoresis gels were analyzed with Quantity One 4.6 analysis software (BioRad Laboratories, Hercules, CA, USA). All solutions in this procedure contained a mixture of protease and phosphatase inhibitors. 


\section{Immunoprecipitation}

Immunoprecipitation was performed following the method described in a previous study [34]. Cell lysates were prepared in NP40 lysis buffer (50 mM Tris- $\mathrm{HCl} \mathrm{pH} \mathrm{7.4,} 150 \mathrm{mM} \mathrm{NaCl}, 0.5 \% \mathrm{NP} 40$, all from Sigma) or high-salt lysis buffer (20 mM HEPES pH 7.4, 10\% glycerol, $0.35 \mathrm{M} \mathrm{NaCl}, 1 \mathrm{mM} \mathrm{MgCl} 2,0.5 \%$ Triton X-100, and $1 \mathrm{mM}$ DTT, all from Sigma-Aldrich) with proteinase inhibitors. The supernatant was then incubated with protein $\mathrm{G}$ beads (GE Healthcare) and the NFATc1 antibody (Abcam, Cambridge, MA, USA) at $4^{\circ} \mathrm{C}$ for 4 h. Beads conjugated with the lysates and antibodies were collected by centrifugation and washed three times with lysis buffer. The final volume of wash buffer was aspirated, and SDS loading buffer was added to the beads. The prepared proteins were resolved using 10\% SDS-PAGE and then transferred to nitrocellulose membranes. Finally, the membranes were incubated with antibodies against Osterix (Abcam, Cambridge, MA, USA) for $12 \mathrm{~h}$. Chemiluminescence was detected using the abovementioned ECL system.

\section{Statistical analysis}

SPSS 17 (SPSS Science, Chicago, IL, USA) was used for data analysis. Quantitative data were expressed as the mean \pm SD. One-way ANOVA followed by Dunnett's post hoc test and Student's $t$ tests were used to analyze differences in the quantitative results. Statistical significance was defined as $P<0.05$.

\section{Results}

\section{Characterization of BMSCs and De-BMSCs}

The general process of osteogenic differentiation and dedifferentiation is schematically illustrated (Fig. 1a). To determine whether BMSCs and De-BMSCs have stem cell characteristics, we used flow cytometry to analyze cell-surface markers. The profiling showed that De-BMSCs expressed the stemness markers CD29 and CD44 but were negative for the hematopoietic markers CD34 and CD45 (Fig. 1b). The cell surface antigen profiles of De-BMSCs were similar to those of BMSCs. To determine whether De-BMSCs have the potential to differentiate into multiple lineages, osteogenic, chondrogenic and adipogenic differentiation was induced. The results demonstrated that De-BMSCs could differentiate into osteoblasts, chondrocytes and adipocytes, which was verified by positive staining for alkaline phosphatase (ALP), safranin 0 , and oil red O, respectively (Fig. 1C). The above results indicated that DeBMSCs retained stem cell properties.

\section{Optimal dedifferentiation culture time to promote the osteogenic differentiation potential of De-BMSCs}

It was reported that De-BMSCs had improved osteogenic potential in vitro and in vivo, and the time for dedifferentiation culture was 7 to 14 days [22,30]. To explore the optimal dedifferentiation culture time to promote the osteogenic differentiation ability of De-BMSCs, we cultured BMSCs in medium with no osteogenic factors for 3, 7, and 10 days after osteogenic induction and then transferred the De-BMSCs to osteogenic medium again for 2 weeks. The osteogenic differentiation ability of the cells was tested. 
Alizarin red S staining revealed that there were significantly more positive calcium nodules formed in the De-BMSCs that were dedifferentiated and cultured for 3 days (Fig. 2a). ALP staining showed similar results (Fig. 2a). We further examined the mRNA and protein expression of osteoblast marker genes after osteogenic differentiation of De-BMSCs cultured for different dedifferentiation times. The results demonstrated that the mRNA expression of COL1A1, OCN and OPN in De-BMSCs subjected to dedifferentiation culture for 3 days was significantly increased (Fig. 2b), and the same trend was also found for protein expression (Fig. 2c). The above results indicated that the optimal dedifferentiation culture time to promote the osteogenic differentiation potential of De-BMSCs was 3 days.

\section{De-BMSC transplantation could promote tendon-bone healing after ACLR}

Tendon-bone healing is an important factor for successful ACLR, and bone formation plays an important role in the process of tendon-bone healing [15]. In view of the enhanced osteogenic differentiation ability of De-BMSCs, we transplanted De-BMSCs that were dedifferentiated and cultured for 3 days at the tendon-bone interface after ACLR. At week 12, the rabbits in each group were sacrificed to evaluate tendon-bone healing by histologic staining, micro-CT examination, and biomechanical testing. $\mathrm{HE}$ staining showed that the interface zone was organized and that perpendicular collagen fibers formed crossing the junction between tendon and bone in the control group; a mature zone of cartilage was observed gradually blending into the adjacent bone and tendon, and tight fibrous tissue and a small amount of osteogenic bone tissue could be seen in the BMSC group; more newly formed surrounding bone was observed in the De-BMSC group, and there was an obvious migration connection between the newly formed bone tissue and bone tunnel (Fig. 3a). Micro-CT analysis showed more new bone formation around the tendon graft in the De-BMSC group than in the other two groups (Fig. 3d), the BV/TV value of the De-BMSC group was significantly higher than those of the control group and BMSCs group (Fig. 3e), and the bone tunnel areas of the De-BMSCs group were smaller than those of the control group (Fig. 3f). To further confirm the formation of bone tissue at the tendon-bone interface, RNA was extracted from the regenerated tissues at the tendon-bone interface of all groups. The mRNA expression of COL1A1, OCN and OPN in the De-BMSC group was significantly increased (Fig. $3 \mathrm{~h}, \mathrm{j}, \mathrm{I}$ ). Immunohistochemical staining of sections with COL1A1, OCN and OPN antibodies showed that the tendon-bone interface stained positive for these proteins. Staining for Col2A1, OCN and OPN was stronger in the De-BMSC group than in the other two groups (Fig. 3g, i, k), which was consistent with the mRNA expression results. To clarify the effect of bone formation on tendon-bone healing after ACLR, we examined the biomechanics of the graft. The maximum failure load values in the Control, BMSCs and De-BMscs groups were $42.01 \pm 6.95 \mathrm{~N}$, $49.24 \pm 3.22 \mathrm{~N}$, and $65.03 \pm 7.96 \mathrm{~N}$, and the stiffness values were $12.39 \pm 1.56 \mathrm{~N} / \mathrm{mm}, 16.26 \pm 2.95$ $\mathrm{N} / \mathrm{mm}, 25.47 \pm 2.58 \mathrm{~N} / \mathrm{mm}$. The maximum failure load and stiffness in the De-BMSC group were higher than those in the other two groups (Fig. 3b, c). These results implied that the transplantation of DeBMSCs could increase bone formation at the tendon-bone interface, thereby promoting tendon-bone healing after ACLR.

\section{Nanog/NFATc1/Osterix expression increased during the osteogenic differentiation of De-BMSCs}


Nanog plays an essential role in maintaining the self-proliferation and undifferentiated state of stem cells [23]. Osterix is the key transcription factor for osteogenic differentiation and can induce the expression of many mature osteoblast marker genes [25]. Nuclear factor of activated T-cells c1 (NFATc1) also plays an important role in bone formation. It combines with Osterix to form a complex and regulate osteoblast differentiation [28]. Therefore, we investigated the expression of Nanog, NFATc1, and Osterix in regenerated tissues at the tendon-bone interface. The results showed that the mRNA and protein expression levels of Nanog (Fig. 4a, b), NFATc1 (Fig. 4c, d) and Osterix (Fig. 4e, f) in the De-BMSC group were significantly higher than those in the other two groups at 12 weeks after ACLR. During the osteogenic differentiation of De-BMSCs, the mRNA and protein expression levels of Nanog (Fig. $4 \mathrm{~g}, \mathrm{~h}$ ), NFATc1 (Fig. 4i, j) and Osterix (Fig. 4k, l) were also obviously increased. This result suggested that the Nanog/NFATc1/Osterix signaling pathway may play an important role in the enhanced osteogenic differentiation ability of De-BMSCs.

\section{The Nanog/NFATc1/Osterix signaling pathway mediated the enhanced osteogenic differentiation ability of De-BMSCs}

To investigate the molecular mechanism of the enhanced osteogenic differentiation ability of De-BMSCs, we performed RNAi-mediated stable knockdown of NFATc1 expression in cultured De-BMSCs. NFATc1 expression was significantly suppressed at both the mRNA and protein levels (Fig. 5a, b). Alizarin red $S$ staining revealed more calcium nodules in the De-BMSC group than in the BMSC group. When si-NFATc1 treatment was performed, the number of stained calcium nodules decreased (Fig. 5c). ALP staining showed a similar result (Fig. 5c). To further verify the essential role of NFATc1 in the observed osteogenic effect, we investigated the expression of osteogenic marker genes. The results demonstrated that the mRNA and protein expression levels of COL1A1 (Fig. 5d, e), OCN (Fig. 5f, g) and OPN (Fig. 5h, i) were increased in De-BMSCs compared with BMSCs during osteogenic differentiation, and the expression of the above genes was significantly decreased when si-NFATC1 was used and lower than that in BMSCs. The above results indicated that NFATc1 plays an important role in the enhanced osteogenic differentiation ability of De-BMSCs. During the differentiation of stem cells, Nanog upregulates the expression of NFATc1, and osteoblast differentiation is regulated by the complex formed by NFATc1 and Osterix. Therefore, we examined the expression of Nanog (Fig. 5j, k), NFATc1(Fig. 5l, m), and Osterix (Fig. $5 n, 0)$. The results showed that the mRNA and protein expression levels of Nanog, NFATc1 and Osterix in De-BMSCs were increased compared with those in BMSCs during osteogenic differentiation. After siNFATc1 administration, the expression of NFATC1 and Osterix decreased significantly and was lower than that in BMSCs, while no significant change was observed in the expression of Nanog (Fig. 5j, k). This suggested that NFATC1 might mediate the enhanced osteogenic differentiation ability of De-BMSCs through the interaction with Osterix. To clarify this mechanism, we used Co-IP to determine whether NFATc1 interacted with Osterix. The results demonstrated that during the osteogenic differentiation of DeBMSCs, the binding of NFATC1 and Osterix was significantly increased compared with that observed during the differentiation of BMSCs, and the interaction of NFATc1 and Osterix was significantly reduced after knocking down NFATc1 (Fig. 5p). The above results indicated that increased Nanog expression upregulated the expression of NFATc1 during the osteogenic differentiation of De-BMSCs, which

Page 11/25 
enhanced the interaction of NFATc1 and Osterix and subsequently increased the expression of Osterix, thereby significantly enhancing the osteogenic differentiation ability.

\section{Discussion}

In the present study, we found that De-BMSCs could retain the characteristics of stem cells and regain the potential for multilineage differentiation. The capacity for osteogenic differentiation was improved in DeBMSCs, as indicated by increased osteoblast marker gene expression. We transplanted De-BMSCs to the tendon-bone interface after ACLR, and the results showed that more newly formed bone was observed at the tendon-bone interface than observed after transplantation of BMSCs; this change improved the biomechanical strength. It was further found that the Nanog/NFATc1/Osterix signaling pathway mediated the enhanced potential for osteogenic differentiation of De-BMSCs.

Tendon-to-bone insertion healing after ACLR is divided into direct healing and indirect healing [35]. During the process of tendon-bone healing, BMSCs infiltrate and are recruited to the interface, and a variety of cytokines are released and promote the proliferation and differentiation of stem cells to achieve tendonbone healing [36]. Considering the role of stem cells in tendon-bone healing, many types of stem cells, such as adipose-derived mesenchymal stem cells [37-38], BMSCs [39-40], induced pluripotent stem cells (iPSCs) [41], umbilical cord stem cells [42], tendon-derived stem cells [43] and CD34+ ACL-derived stem cells [44], have been used to promote tendon-bone healing after ACLR. Among these stem cells, BMSCs are recognized as having the best proliferation and differentiation potential [45]. Therefore, there are many studies on the application of BMSCs to promote tendon-bone healing, and the results showed that transplantation of BMSCs could promote tendon bone healing after ACL reconstruction, mainly indicated by increased cartilage formation at the tendon-bone interface [39-40]. The strength of the tendon-to-bone attachment correlated with new bone formation around the tendon [46]. Although the application of BMSCs can promote healing of the tendon-bone interface, insufficient new bone formation may affect the early biomechanical strength after reconstruction.

Studies have shown that the osteogenic differentiation ability of De-BMSCs in vitro and the bone formation of De-BMSCs in vivo were increased [23,30], but research on the application of De-BMSCs in tendon-bone healing after ACLR has not been reported. To our knowledge, this is the first report of the use of De-BMSCs to enhance bone formation at the tendon-bone interface. Micro-CT scans showed that there was more new bone generation around the graft in the De-BMSCs group than in the BMSCs group. The results of biomechanical testing showed that the maximum load and stiffness of the De-BMSC group were both higher than those of the BMSC group. These results implied that transplantation of De-BMSCs could increase new bone formation at the tendon-bone interface and enhance biomechanical strength after ACLR. Lim et al. [38] and Soon et al. [39] used BMSCs to promote tendon-bone healing after ACLR with autograft and allograft tendons, respectively. The biomechanical strength of the BMSC group in our study was similar to that reported in the abovementioned studies, and the biomechanical strength of DeBMSCs group was higher than that of BMSCs group in this study. Therefore, transplantation of DeBMSCs could increase bone formation at the tendon-bone interface after ACLR and enhance the 
biomechanical strength of the reconstructed ligament, which was helpful for early postoperative rehabilitation.

Although stem cells have been widely used in various regenerative medicine studies, the low cell survival rate and differentiation efficiency in vivo after transplantation has significantly reduced the effectiveness of stem cell therapy. Studies have shown that the proliferation and differentiation efficiency of De-BMSCs in vitro and in vitro is higher than that of BMSCs $[23,30]$, and the mechanism is mainly due to increased Nanog expression. In the present study, we also found that the expression of Nanog in De-BMSCs was increased, and its expression at the tendon-bone interface also increased after De-BMSC transplantation. Nanog is the key transcription factor controlling MSCs identity and fate conversion and plays a vital role in maintaining the self-proliferation and undifferentiated state of stem cells [24]. Nanog also plays a major role in the increased capacity for osteogenic differentiation in De-BMSCs [23], but the molecular mechanism is still unclear. Osterix is a zinc finger-containing osteoblast-specific transcription factor that can induce the expression of many mature osteoblast genes. It has been confirmed to be involved in osteoblast differentiation, maturation and activity [47] and is also necessary for the maturation and function of osteocytes postnatally [48]. Osteogenic differentiation is a key step in osteogenesis, so Osterix also plays an important role in the process of osteogenic differentiation of stem cells. Our study showed that the expression of Osterix increased during the osteogenic differentiation of BMSCs and DeBMSCs. NFATc1 is an important transcription factor for osteoclast formation [49], and it can induce osteoclast differentiation and promote bone resorption. However, some studies have shown that the NFAT signaling pathway also plays a role in the differentiation of osteoblasts, the consequences of NFAT signaling in osteoblastic cells are controversial, and both stimulatory and inhibitory effects on osteoblastic differentiation have been reported [50]. The results of our study showed that the expression of NFATc1 was higher during the osteogenic differentiation of De-BMSCs than during that of BMSCs. The cooperation of NFATc1 and Osterix plays an important role in the formation of new bone [51]. In the present study, we found that NFATc1 interacted with Osterix during the osteogenic differentiation of BMSCs; the interaction between NFATc1 and Osterix was significantly enhanced in the osteogenic differentiation of De-BMSCs, and the expression of osteoblast marker genes was obviously increased. When siRNA was used to knock down the expression of NFATc1, the interaction between NFATc1 and Osterix was reduced, and the expression of osteoblast marker genes was decreased accordingly. During the process of osteogenic differentiation of stem cells, Nanog could promote osteogenic differentiation by enhancing the interaction between NFATc1 and Osterix, and the increased NFATc1 expression induced by Nanog might play an important role [52]. Our results demonstrated that the expression of Nanog was increased in De-BMSCs compared with BMSCs and that the expression of NFATc1 was also elevated during the osteogenic differentiation of De-BMSCs. Therefore, during the process of osteogenic differentiation of De-BMSCs, the increased Nanog expression enhanced the binding of NFATc1 and Osterix by upregulating NFATc1 and promoted the expression of Osterix at the same time; the expression of downstream osteoblast marker genes was increased accordingly, thereby enhancing the osteogenic differentiation ability of dedifferentiated BMSCs. 


\section{Conclusions}

In summary, our study found that the osteogenic differentiation ability of De-BMSCs was significantly enhanced. The mechanism was that the Nanog/NFATc1/Osterix signaling pathway increased the expression of osteoblast marker genes. Transplantation of De-BMSCs after ACLR could increase bone formation at the tendon-bone interface, thereby increasing the biomechanical strength of the reconstructed ligament. This study provides a new method for promoting tendon-bone healing after ACLR and offers new insight into the clinical application of stem cells.

\section{Abbreviations}

MSCs: Mesenchymal stem cells; BMSCs: bone marrow mesenchymal stem cells; De-BMSCs: differentiated osteogenic bone marrow mesenchymal stem cells Col1A1: 1 chain of type I collagen; OCN: osteocalcin; NFATc1: Nuclear factor of activated T-cells c1; OPN: osteopontine; DMEM: dulbecco's minimum essential medium; RT-PCR: polymerase chain reaction; qRT-PCR: Quantitative real-time polymerase chain reaction; GAPDH: glyceraldehyde 3-phosphate dehydrogenase; EDTA: ethylene diamine tetraacetic acid; SDS-PAGE: sodium-dodecyl sulfate-polyacrylamide gel electrophoresis; HEPES: N-2hydroxyethylpiperazine-N-ethane-sulphonicacid.

\section{Declarations}

\section{Acknowledgments}

The authors thank the staff of the Center for Animal Experiments of Zhongnan Hospital of Wuhan University

\section{Funding}

This work was supported by the National Natural Science Foundation of China (Nos. 81702159 and 81972036).

\section{Availability of data and material}

The datasets used and/or analyzed during the current study are available from the corresponding author on reasonable request.

\section{Authors' contributions}

Conception and design: LBC and HW. Acquisition of data: KT, JHC, HX and YFSG. Analysis and interpretation of data: KT, JQ, HX. Drafting the manuscript: KT, JHC, LBC and HW. Revising the manuscript: LBC and HW. All authors read and approved the final manuscript.

\section{Ethics approval}


All animal experiments were performed in accordance with the Guidelines for the Care and Use of Laboratory Animals from the National Research Council of the United States National Academies, and approved by the Committee on the Ethics of Animal Experiments of the Wuhan University School of Medicine.

\section{Consent for publication}

Not applicable.

\section{Competing interests}

The authors declare that they have no competing of interests.

\section{References}

[1] Goldstein J, Bosco JA 3rd. The ACL-deficient knee: natural history and treatment options. Bull Hosp Jt Dis. $2001 ; 60: 173-8$.

[2] Beynnon BD, Johnson RJ, Abate JA, Fleming BC, Nichols CE. Treatment of anterior cruciate ligament injuries, part I. Am J Sports Med. 2005; 33:1579-1602.

[3] Fu FH, Bennett CH, Ma CB, Menetrey J, Lattermann C. Current trends in anterior cruciate ligament reconstruction: part II. Operative procedures and clinical correlations. Am J Sports Med. 2000;28(1): 12430.

[4] Salmon LJ, Russell VJ, Refshauge K, Kader D, Connolly C, Linklater J, Pinczewski LA. Long-term outcome of endoscopic anterior cruciate ligament reconstruction with patellar tendon autograft: minimum 13-year review. Am J Sports Med. 2006;34(5):721-32

[5] Mall NA, Chalmers PN, Moric M, Tanaka MJ, Cole BJ, Bach BR Jr, Paletta GA Jr. Incidence and trends of anterior cruciate ligament reconstruction in the United States. Am J Sports Med. 2014;42(10):2363-70.

[6] Duchman KR, Lynch TS, Spindler KP. Graft selection in anterior cruciate ligament surgery: who gets what and why? Clin Sports Med. 2017;36(1):25-33.

[7] Ruffilli A, Traina F, Evangelisti G, Borghi R, Perna F, Faldini C. Preservation of hamstring tibial insertion in anterior cruciate ligament reconstruction: a review of the current literature. Musculoskelet Surg. 2015;99(2):87-92.

[8] Kraeutler MJ, Welton KL, McCarty EC, Bravman JT. Revision Anterior Cruciate Ligament Reconstruction. J Bone Joint Surg Am. 2017 Oct 4;99(19):1689-96.

[9] Kanazawa T, Soejima T, Noguchi K, Tabuchi K, Noyama M, Nakamura K, Shiba N. Tendon-to-bone healing using autologous bone marrow-derived mesenchymal stem cells in ACL reconstruction without a 
tibial bone tunnel. A histological study. Muscles Ligaments Tendons J. 2014;4(2):201-6.

[10] Andriolo L, Di Matteo B, Kon E, Filardo G, Venieri G, Marcacci M. PRP Augmentation for ACL Reconstruction. Biomed Res Int. 2015; 2015:371746.

[11] Chen B, Li B, Qi Y, Ni Q, Pan Z, Wang H, Chen L. Enhancement of tendon-to-bone healing after anterior cruciate ligament reconstruction using bone marrow- derived mesenchymal stem cells genetically modified with bFGF/ BMP2. Sci Rep. 2016;6(1):25940.

[12] Li YG, Wei JN, Lu J, Wu XT, Teng GJ. Labeling and tracing of bone marrow mesenchymal stem cells for tendon-to-bone tunnel healing. Knee Surg Sports Traumatol Arthrosc. 2011; 19(12):2153-8.

[13] Hao ZC, Wang SZ, Zhang XJ, Lu J. Stem cell therapy: A promising biological strategy for tendon-bone healing after anterior cruciate ligament reconstruction. Cell Prolif. 2016; 49:154-62.

[14] Figueroa D, Calvo R, Vaisman A, Arellano S, Figueroa F, Donoso R, Bernal N, O'Connell L. Arthroscopic Intercondylar Notch Bone Marrow Aspiration During Anterior Cruciate Ligament Reconstruction. Arthrosc Tech. 2019;8(12): e1437-41.

[15] Lui PP区Ho G $₫$ Shum WT囚Lee YW, Ho PY, Lo WN, Lo CK. Inferior tendon graft to bone tunnel healing at the tibia compared to that at the femur after anterior cruciate ligament reconstruction. J Orthop Sci. 2010; 15(3):389-401.

[16] Wen CY, Qin L, Lee KM, Chan KM. Peri-graft bone mass and connectivity as predictors for the strength of tendon-to-bone attachment after anterior cruciate ligament reconstruction. Bone. 2009; 45(3):545-52.

[17] Soon M, Hassan A, Hui J, Goh J, Lee E. An analysis of soft tissue allograft anterior cruciate ligament reconstruction in a rabbit model: a short-term study of the use of mesenchymal stem cells to enhance tendon osteointegration. Am J Sports Med. 2007;35(6): 962-71.

[18] Lim JK, Hui J, Li L, Thambyah A, Goh J, Lee EH. Enhancement of tendon graft osteointegration using mesenchymal stem cells in a rabbit model of anterior cruciate ligament reconstruction. Arthroscopy. 2004; 20(9): 899-910.

[19] Jopling C, Boue S, Belmonte J. Dedifferentiation, transdifferentiation and reprogramming: three routes to regeneration. Nat Rev Mol Cell Biol. 2011;12(2): 79-89.

[20] Jiang F, Feng Z, Liu H, Zhu J. Involvement of plant stem cells or stem cell-like cell in dedifferentiation. Front Plant Sci. 2015; 18; 6:1028.

[21] Liu Y, Jiang X, Zhang X, Chen R, Sun T, Fok KL, Dong J, Tsang LL, Yi S, Ruan Y, Guo J, Yu MK, Tian Y, Chung YW, Yang M, Xu W, Chung CM, Li T, Chan HC. Dedifferentiation-reprogrammed mesenchymal stem cells with improved therapeutic potential. Stem Cells. 2011; 29(12):2077-89. 
[22] Rui Y, Xu L, Chen R, Zhang T, Lin S, Hou Y, Liu Y, Meng F, Liu Z, Ni M, Tsang KS, Yang F, Wang C, Chan HC, Jiang X, Li G. Epigenetic memory gained by priming with osteogenic induction medium improves osteogenesis and other properties of mesenchymal stem cells. Sci Rep. 2015; 5:11056.

[23] Go MJ, Takenaka C, Ohgushi H. Forced expression of Sox2 or Nanog in human bone marrow derived mesenchymal stem cells maintains their expansion and differentiation capabilities. Exp Cell Res. 2008; 314(5):1147-54

[24] Nakashima K, Zhou X, Kunkel G, Zhang Z, Deng JM, Behringer RR, de Crombrugghe B. The novel zincfinger-containing transcription factor Osterix is required for osteoblast differentiation and bone formation. Cell. 2002;108:17-29.

[25] Kaback LA, Soung do Y, Naik A, Smith N, Schwarz EM, O'Keefe RJ, Drissi H. Osterix regulates mesenchymal stem cell mediated endochondral ossification. J Cell Physiol. 2008; 214(1):173-82.

[26] Liu Q, Li M, Wang S, Xiao Z, Xiong Y, Wang G. Recent Advances of Osterix Transcription Factor in Osteoblast Differentiation and Bone Formation. Front Cell Dev Biol. 2020; 8: 601224.

[27] Kim K囚Kim JH囚Lee J囚Jin HM, Lee SH, Fisher DE, Kook H, Kim KK, Choi Y, Kim N.Nuclear factor of activated T cells $\mathrm{c} 1$ induces osteoclast- associated receptor gene expression during tumor necrosis factor-related activation-induced cytokine-mediated osteoclastogenesis. J Biol Chem. 2005; 280(42):35209-16.

[28] Koga T, Matsui Y, Asagiri M, Kodama T, de Crombrugghe B, Nakashima K, Takayanagi H. NFAT and Osterix cooperatively regulate bone formation. Nat Med. 2005; 11(8):880-5.

[29] Ogasawara T, Ohba S, Yano F, Kawaguchi H, Chung UI, Saito T, Yonehara Y, Nakatsuka T, Mori Y, Takato T, HoshiK. Nanog promotes osteogenic differentiation of the mouse mesenchymal cell line C3H10T1/2 by modulating bone morphogenetic protein (BMP) signaling. J Cell Physiol. 2013; 228(1):163-71.

[30] Fu X, Yan H, Zhang H, Wang G, Liu K, Gu Q, Tao Y, Chen G, Jiang X, Li G, Gu Y, Shi Q. Improved osteogenesis and upregulated immunogenicity in human placenta-derived mesenchymal stem cells primed with osteogenic induction medium. Stem Cell Res Ther. $2016 ; 7(1): 138$.

[31] Tie K, Wu M, Deng Y, Wen Y, Dan Xu, Chen L, Wang H. Histone hypo-acetylation of Sox9 mediates nicotine-induced weak cartilage repair by suppressing BMSC chondrogenic differentiation. Stem Cell Res Ther. 2018; 9(1):98.

[32] Liu S, Sun Y, Wan F, Ding Z, Chen S, Chen J. Advantages of an Attached Semitendinosus Tendon Graft in Anterior Cruciate Ligament Reconstruction in a Rabbit Model. Am J Sports Med. 2018; 46(13): 3227-36. 
[33] Sasaki K, Kuroda R, Ishida K, Kubo S, Matsumoto T, Mifune Y, Kinoshita K, Tei K, Akisue T, Tabata Y, Kurosaka M. Enhancement of tendon-bone osteointegration of anterior cruciate ligament graft using granulocyte colony-stimulating factor. Am J Sports Med. 2008;36(8):1519-27.

[34] Su SK, Li CY, Lei PJ, Wang X, Zhao QY, Cai Y, Wang Z, Li L, Wu M. The EZH1-SUZ12 complex positively regulates the transcription of NF-KB target genes through interaction with UXT. J Cell Sci. 2016; 129: $2343-53$.

[35] M Benjamin, H Toumi, J R Ralphs, G Bydder, T M Best, S Milz. Where tendons and ligaments meet bone: attachment sites ('entheses') in relation to exercise and/or mechanical load. J Anat. 2006; 208(4): 471-90.

[36] Ppy Lui, P Zhang, Km Chan, L Qin. Biology and augmentation of tendon-bone insertion repair. J Orthop Surg Res. 2010; 5: 59.

[37] Teuschl A, Heimel P, Nürnberger S, Griensven M, Redl H, Nau T. A Novel Silk Fiber-Based Scaffold for Regeneration of the Anterior Cruciate Ligament: Histological Results From a Study in Sheep. Am J Sports Med. 2016; 44(6): 1547-57.

[38] Kosaka M, Nakase J, Hayashi K, Tsuchiya H. Adipose-Derived Regenerative Cells Promote TendonBone Healing in a Rabbit Model. Arthroscopy 2016; 32: 851- 9.

[39] Lim JK, Hui J, Li L, Thambyah A, Goh J, Lee EH. Enhancement of tendon graft osteointegration using mesenchymal stem cells in a rabbit model of anterior cruciate ligament reconstruction. Arthroscopy. 2004; 20(9): 899-910.

[40] Soon MY, Hassan A, Hui JH, Goh J, Lee EH. An analysis of soft tissue allograft anterior cruciate ligament reconstruction in a rabbit model: a short-term study of the use of mesenchymal stem cells to enhance tendon osteointegration. m J Sports Med. 2007; 35(6): 962-71.

[41] Kouroupis D, Kyrkou A, Triantafyllidi E, et al. Generation of stem cell-based bioartificial anterior cruciate ligament (ACL) grafts for effective ACL rupture repair. Stem Cell Res (Amst) 2016; 17: 448-457.

[42] Jang KM, Lim HC, Jung WY, Moon SW, Wang JH. Efficacy and safety of human umbilical cord bloodderived mesenchymal stem cells in anterior cruciate ligament reconstruction of a rabbit model: new strategy to enhance tendon graft healing. Arthroscopy. 2015; 31(8): 1530-9.

[43] Lui PP, Wong OT, Lee YW. Application of tendon-derived stem cell sheet for the promotion of graft healing in anterior cruciate ligament reconstruction. Am J Sports Med. 2014; 42 (3): 681- 9.

[44] Mifune Y, Matsumoto T, Takayama K, Terada S, Sekiya N, Kuroda R, Kurosaka M, Fu FH, Huard J. Tendon graft revitalization using adult anterior cruciate ligament (ACL)-derived CD34+ cell sheets for ACL reconstruction. Biomaterials. 2013; 34(22): 5476-87. 
[45] Van Eijk F, Saris DB, Riesle J, Willems WJ, Van Blitterswijk CA, Verbout AJ, Dhert WJ. Tissue engineering of ligaments: a comparison of bone marrow stromal cells, anterior cruciate ligament, and skin fibroblasts as cell source. Tissue Eng. 2004;10(5-6):893-903.

[46] K Anderson, A M Seneviratne, K Izawa, B L Atkinson, H G Potter, S A Rodeo. Augmentation of tendon healing in an intraarticular bone tunnel with use of a bone growth factor. Am J Sports Med. 2001; 29(6): $689-98$.

[47] Ramazzotti G, Fiume R, Chiarini F, Campana G, Ratti S, Billi AM, Manzoli L, Follo MY, Suh PG, McCubrey J, Cocco L, Faenza I. Phospholipase C- $\beta 1$ interacts with cyclin E in adipose- derived stem cells osteogenic differentiation. Adv Biol Regul. 2019; 71: 1-9.

[48] Klein-Nulend J, Bakker AD, Bacabac RG, Vatsa A, Weinbaum S. Mechanosensation and transduction in osteocytes. Bone. 2013; 54(2):182-90.

[49] Ono T, Nakashima T. Recent advances in osteoclast biology. Histochem Cell Biol. 2018;149(4):32541.

[50] Smerdel-Ramoya A, Zanotti S, Canalis E. Connective tissue growth factor (CTGF) transactivates nuclear factor of activated T-cells (NFAT) in cells of the osteoblastic lineage. J Cell Biochem. 2010;110(2):477-83.

[51] Koga T, Matsui Y, Asagiri M, Kodama T, de Crombrugghe B, Nakashima K, Takayanagi H. NFAT and Osterix cooperatively regulate bone formation. Nat Med. 2005; 11(8):880-5.

[52] Ogasawara T, Ohba S, Yano F, Kawaguchi H, Chung UI, Saito T, Yonehara Y, Nakatsuka T, Mori Y, Takato T, HoshiK. Nanog promotes osteogenic differentiation of the mouse mesenchymal cell line C3H10T1/2 by modulating bone morphogenetic protein (BMP) signaling. J Cell Physiol. 2013; 228(1):163-71.

\section{Figures}


a Differentiation

Dedifferentiation Redifferentiation

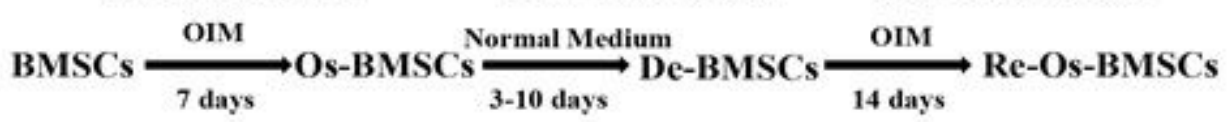

$\mathrm{b}$

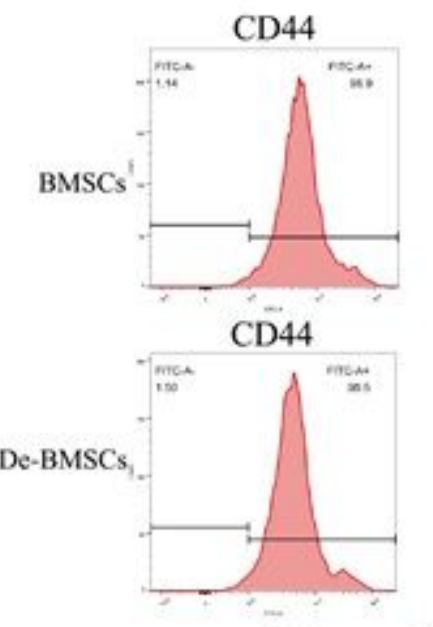

$\mathrm{c}$

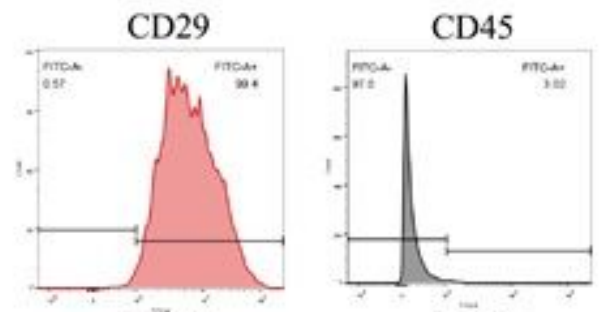

CD29
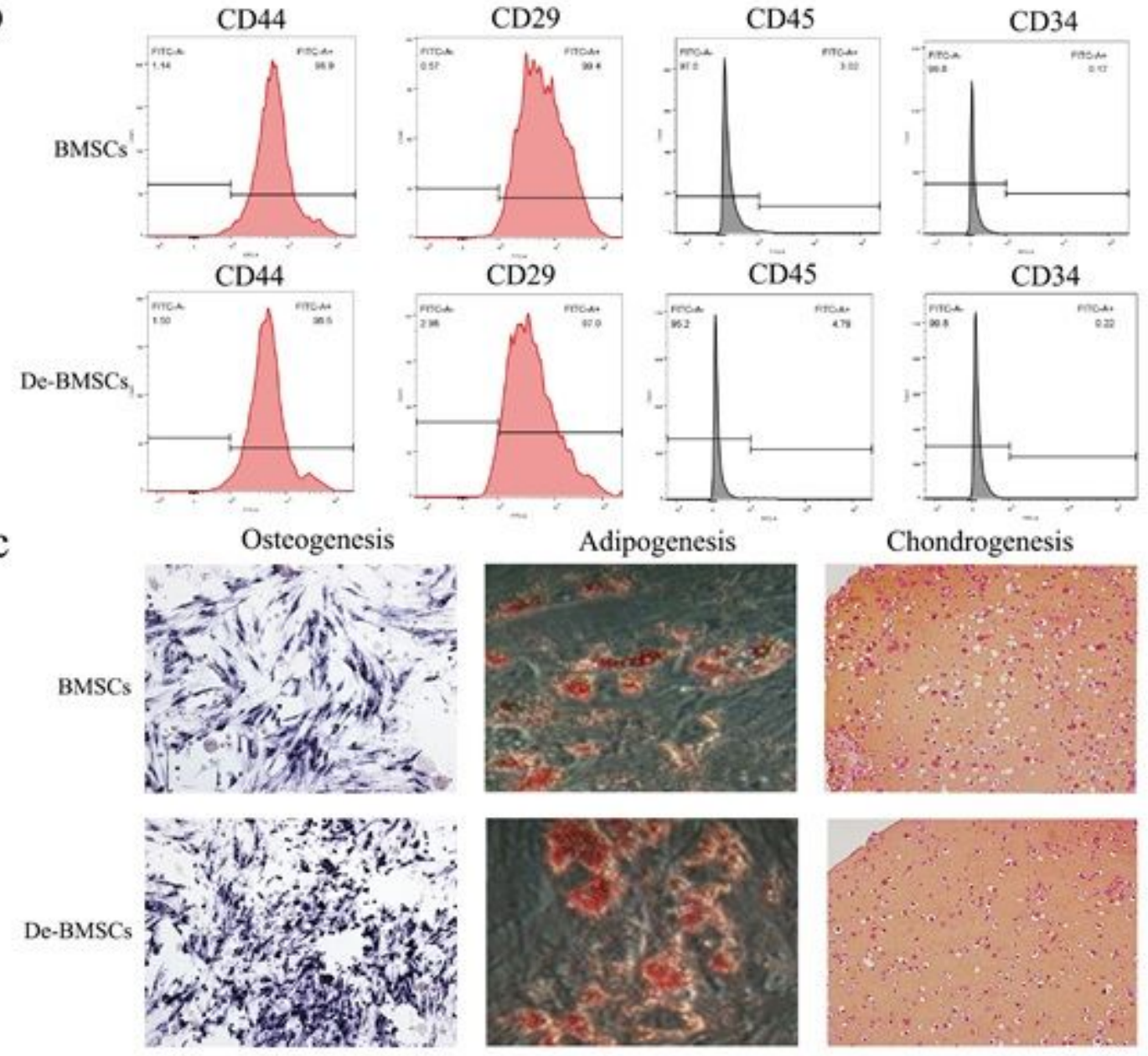

\section{Figure 1}

Characterization of rabbit-derived BMSCs and De-BMSCs. a Schematic diagram illustrating the procedure for generating De-BMSCs. BMSCs that underwent osteogenic differentiation, dedifferentiation and redifferentiation are shown. b Cell surface markers of BMSCs and De-BMSCs. c Alkaline phosphatase (ALP), oil red $O$ and safranin $O$ staining to detect osteogenesis (40x), adipogenesis (100x) and chondrogenesis (100x), respectively, of BMSCs and De-BMSCs. OIM, osteogenic induction media; BMSCs, bone marrow mesenchymal stem cells; Os-BMSCs, osteogenic bone marrow mesenchymal stem cells; DeBMSCs, dedifferentiated osteogenic bone marrow mesenchymal stem cells; Re-Os-BMSCs, redifferentiation osteogenic bone marrow mesenchymal stem cell 


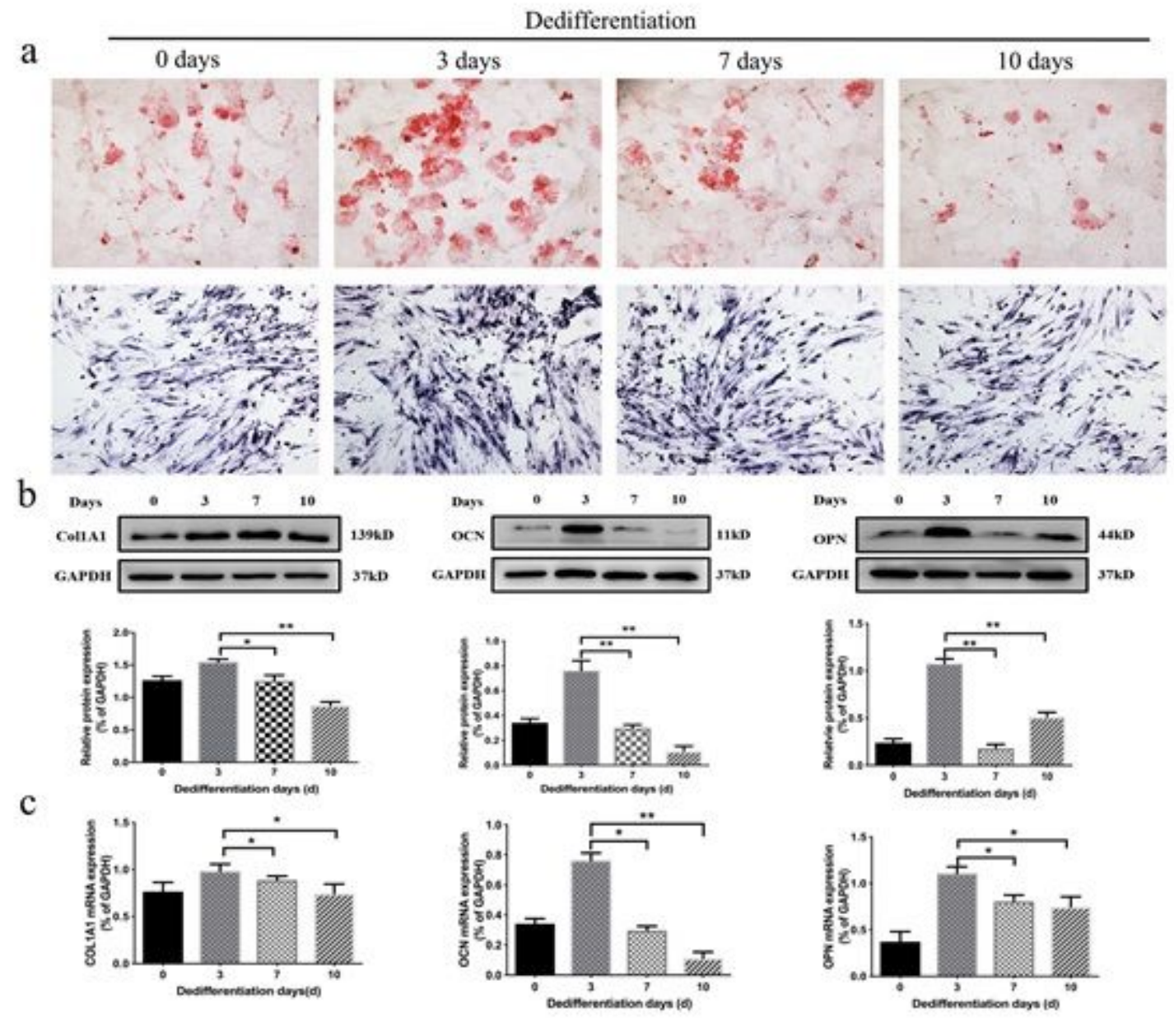

Figure 2

The osteogenic differentiation ability of De-BMSCs cultured for different dedifferentiation times. a Staining with alizarin red S (100x) and staining for alkaline phosphatase (40x) to detect the osteogenic differentiation of De-BMSCs. b mRNA expression of osteoblast marker genes, including COL1A1, OCN and OPN. c Protein expression of osteoblast marker genes, including COL1A1, OCN and OPN. Data represent the mean $\pm S D(n=3) .{ }^{*} p<0.05,{ }^{*} p<0.01$. BMSCs, bone marrow mesenchymal stem cells; DeBMSCs, dedifferentiated osteogenic bone marrow mesenchymal stem cells; COL1A1, a1 chain of type I collagen; OCN, osteocalcin; OPN, osteopontin 

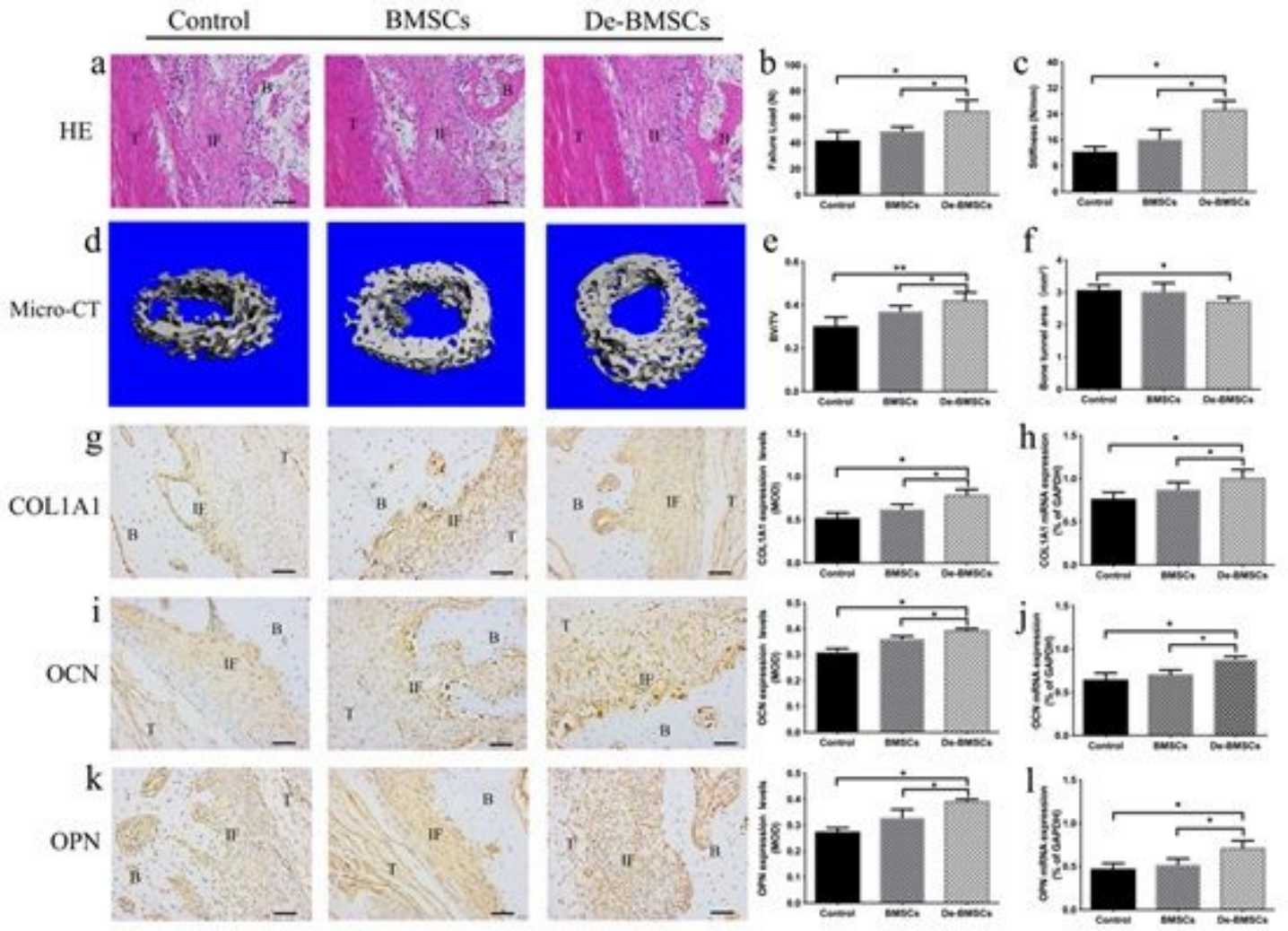

Figure 3

Tendon-bone healing 12 weeks after anterior cruciate ligament reconstruction (ACLR). a Hematoxylin and eosin staining of the tendon-bone interface. b Maximum failure load. c Stiffness. d Microcomputed tomography evaluation of the tendon-bone interface. e Bone volume/total volume (BV/TV). $f$ Bone tunnel area. $\mathrm{g}$ Immunohistochemical staining for COL1A1 and quantification of the MOD at the tendon-bone interface. $\mathrm{h}$ mRNA expression of COL1A1 at the tendon-bone interface. i Immunohistochemical staining for OCN and quantification of the MOD at the tendon-bone interface. j mRNA expression of OCN at the tendon-bone interface. $\mathrm{k}$ Immunohistochemical staining for OPN and quantification of the MOD at the tendon-bone interface. I mRNA expression of OPN at the tendon-bone interface. Data represent the mean $\pm S D(n=5) .{ }^{*} p<0.05,{ }^{\star} p p<0.01$. Scale bar $=50 \mu \mathrm{m}$. BMSCs, bone marrow mesenchymal stem cells; DeBMSCs, differentiation bone marrow mesenchymal stem cells; COL1A1, a1 chain of type I collagen; OCN, osteocalcin; OPN, osteopontin; MOD, mean of density; B, bone; IF, interface; T, tendon 


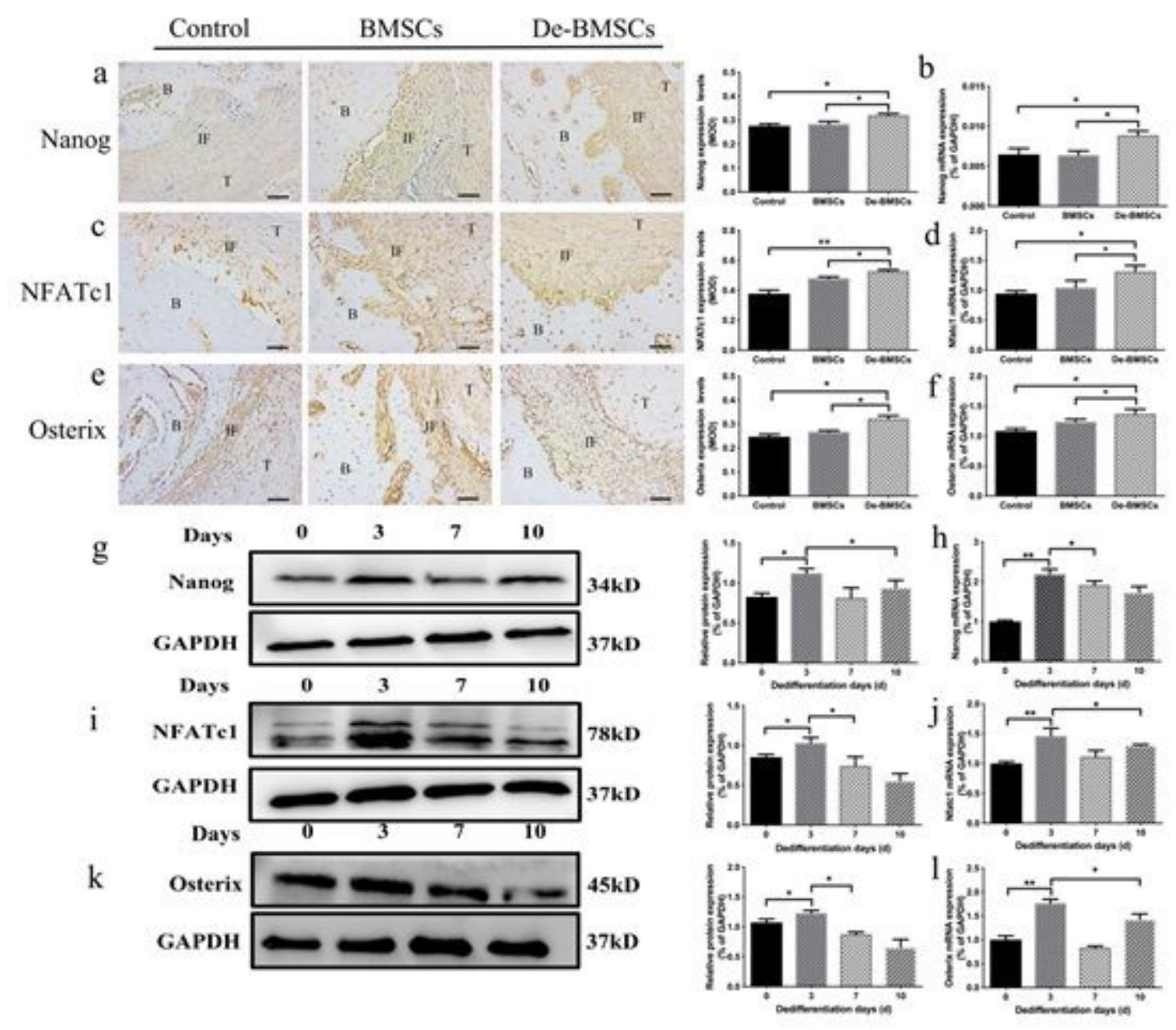

Figure 4

Expression of Nanog/NFATc1/Osterix signaling pathway components at the tendon-bone interface at 12 weeks after anterior cruciate ligament reconstruction (ACLR). a Immunohistochemical staining for Nanog and quantification of the MOD at the tendon-bone interface. b mRNA expression of Nanog at the tendonbone interface. $c$ Immunohistochemical staining for NFATc1 and quantification of the MOD at the tendonbone interface. $\mathrm{d}$ mRNA expression of NFATc1 at the tendon-bone interface. e Immunohistochemical staining for Osterix and quantification of the MOD at the tendon-bone interface. $f$ mRNA expression of Osterix at the tendon-bone interface. g Protein expression of Nanog. h mRNA expression of Nanog. i Protein expression of NFATc1. j mRNA expression of NFATc1. $k$ Protein expression of Osterix. I mRNA expression of Osterix. Data represent the mean $\pm S D\left(n=5\right.$ in vivo, $n=3$ in vitro). Scale bar $=50 \mu \mathrm{m}$. ${ }^{*}<$ $0.05,{ }^{\star \star} p<0.01$. BMSCs, bone marrow mesenchymal stem cells; De-BMSCs, dedifferentiated osteogenic bone marrow mesenchymal stem cells; NFATc1, nuclear factor of activated T-cells c1; B, bone; IF, interface; $T$ tendon 


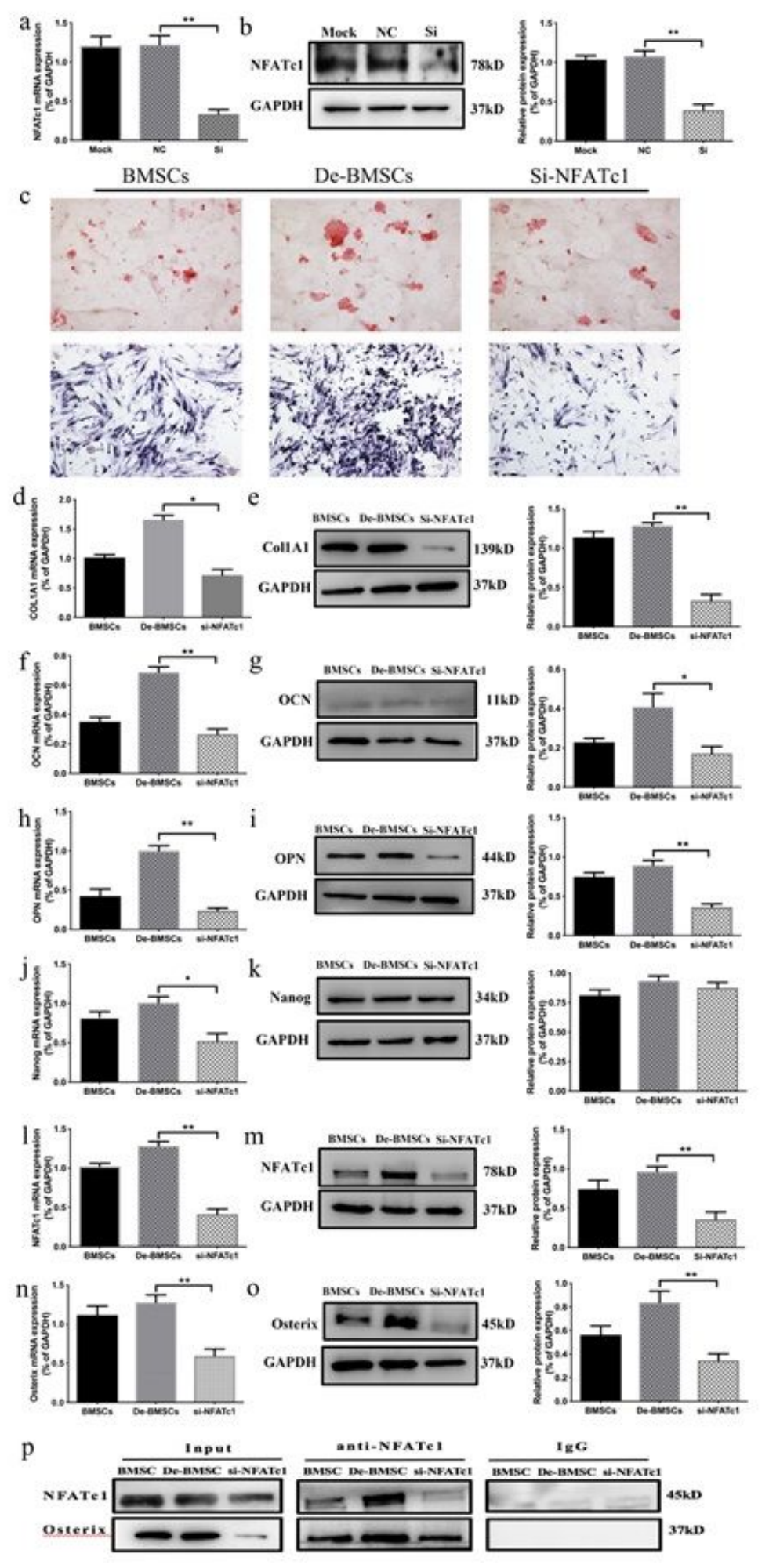

\section{Figure 5}

Mechanism underlying the enhanced osteogenic differentiation ability of De-BMSCs. a and b Effect of SiNFATc1 in De-BMSCs. c Staining with alizarin red S and staining for alkaline phosphatase to detect the osteogenic differentiation of BMSCs and De-BMSCs. d mRNA expression of COL1A1. e Protein expression of COL1A1. f mRNA expression of OCN. g Protein expression of OCN. h mRNA expression of OPN. i Protein expression of OPN. j mRNA expression of Nanog. k Protein expression of Nanog. I mRNA 
expression of NFATc1. m Protein expression of NFATc1. $n$ mRNA expression of Osterix. o Protein expression of Osterix. p Effects of Si-NFATc1 on the interaction of NFATc1 and Osterix. Data represent the mean $\pm S D(n=3) .{ }^{\star} p<0.05,{ }^{*} p<0.01$. BMSCs, bone marrow mesenchymal stem cells; De-BMSCs, dedifferentiated osteogenic bone marrow mesenchymal stem cells; COL1A1, a1 chain of type I collagen; OCN, osteocalcin; OPN, osteopontin; NFATc1, nuclear factor of activated T-cells c1 